\title{
Ankylosing spondylitis associated with primary aldosteronism in a middle-aged woman
}

Young Sun Suh, Hyun-Ok Kim, Yun-Hong Cheon, Wonyong Jo, Jeongmin Hong, and Sang-Il Lee

Division of Rheumatology, Department of Internal Medicine, Gyeongsang National University School of Medicine, Jinju, Korea

Received: April 26, 2015

Revised : June 14, 2015

Accepted: July 10, 2015

\section{Correspondence to}

Sang-Il Lee, M.D.

Division of Rheumatology,

Department of Internal Medicine and Institute of Health Science, Gyeongsang National University School of Medicine, 15 Jinju-daero 816beon-gil, Jinju 52727, Korea

Tel: $+82-55-750-8853$

Fax: +82-55-758-9122

E-mail: goldgu@gnu.ac.kr

\section{To the Editor,}

Primary aldosteronism is the main cause of secondary hypertension. This syndrome is characterized by hypertension, hypokalemia, suppressed plasma renin activity (PRA), and increased aldosterone excretion. Both experimental and clinical data indicate that aldosterone acts as a mineralocorticoid hormone involved in regulating electrolyte balance and volume homeostasis and can promote inflammatory damage to target organs [1]. Therefore, these proinflammatory actions induced by aldosterone may contribute to chronic inflammatory autoimmune diseases.

Ankylosing spondylitis (AS) is a chronic inflammatory disease that can involve the spine and sacroiliac joint and is associated with high expression of HLA B27 [2]. AS was thought to be a disease that almost exclusively affected young men, rarely occurring in middle-aged women. Moreover, the coexistence of primary aldosteronism and AS has previously not been described. Recently, we treated a patient with primary aldosteronism who developed AS and significantly improved with treatment consisting of spironolactone and a tumor necrosis factor $\alpha$ (TNF- $\alpha$ ) inhibitor. Thus, we report a case and review of the literature on coexisting primary aldosteronism and AS in a middle-aged woman.

A 59-year-old female, who had a 20- year history of hypertension and was receiving antihypertensive medication, had worsening hypertension for the previous 2 months. One month earlier, she was admitted to another hospital with chest pain. Her chest radiography showed mild cardiomegaly, and auscultation of the heart and lungs was normal. Cardiac tests were normal, but thyrotoxicosis and hypokalemia were found. Her serum potassium and blood pressure remained uncorrected despite oral potassium supplementation and antihypertensive medications. Therefore, she was referred to our hospital to determine the cause of her refractory arterial hypertension and hypokalemia.

On admission, her blood pressure was $164 / 83 \mathrm{mmHg}$, pulse rate 70 per minute, body temperature $36^{\circ} \mathrm{C}$, respiration rate 16 breaths per minute, body mass index $24.0 \mathrm{~kg} / \mathrm{m}^{2}$, and she had no edema. Laboratory analysis showed hypernatremia (146.3 mEq/L; reference range, 135.0 to 145.0) and hypokalemia (2.7 mEq/L; reference range, 3.5 to 5.5). Based on these findings, primary aldosteronism was highly suspected. Subsequent investigation showed a suppressed PRA $(<0.10$ $\mathrm{ng} / \mathrm{mL} / \mathrm{hr}$; reference range, 0.15 to 2.33), high plasma aldosterone concentration (PAC $39.5 \mathrm{ng} / \mathrm{dL}$; normal value, 1.3 to $14.5 \mathrm{pg} / \mathrm{mL}$ ), and positive PAC/PRA ratio (395 ng/dL:ng/mL/hr; normal value < 30). The saline infusion test revealed an unsuppressed plasma aldosterone level 
( $343.7 \mathrm{ng} / \mathrm{dL}$; normal value < 5.0), and we confirmed primary aldosteronism. Computed tomography (CT) demonstrated two $2 \mathrm{~cm}$ nodular lesions in the right adrenal gland and a suspicious small nodular lesion in the left adrenal gland (Fig. 1). A $1 \mathrm{mg}$ dexamethasone suppression test, urinary free cortisol, and diurnal urinary excretion of metanephrines, vanillylmandelic acid, epinephrine, norepinephrine and dopamine were normal. Hence, we could rule out Cushing syndrome and pheochromocytoma. Then, bilateral adrenal vein sampling (AVS) was performed. The aldosterone concentration was $45,900 \mathrm{pg} /$ $\mathrm{mL}$ in the right adrenal vein and $6,390 \mathrm{pg} / \mathrm{mL}$ in the left adrenal vein. The dominant and non-dominant aldosterone-cortisol (A/C) ratios were greater than the inferior vena cava A/C ratio. Thus, AVS was considered successful, and she had a cortisol-corrected PAC lateralization ratio $<4$.0. Therefore, we diagnosed her with bilateral adrenal hyperplasia. Subsequently, her blood pressure and serum potassium normalized with amlodipine $5 \mathrm{mg}$ and spironolactone $50 \mathrm{mg}$. At the time of admission to our hospital, she had a normal thyroid stimulating hormone (TSH) and elevated free T4. However, serum thyroglobulin, antithyroid peroxidase and TSH receptor antibodies were all negative, and radioactive iodine uptake was only $3 \%$ even in a thyrotoxic state. These results may represent a transient thyrotoxicosis due to the inflammatory effect of excessive aldosterone and normalized with treatment for primary aldosteronism.

During the medical follow-up, spironolactone was tapered to $12.5 \mathrm{mg}$ over 2 years. At that time, she complained of increasing lower back and knee pain with recurrence of hypokalemia. She had had intermittent low back pain for the past 10 years. She described chronic inflammatory lower back pain, morning stiffness lasting for more than 1 hour, improvement with exercise, arthritis of the knee joint, and two exacerbations of anterior uveitis for 6 months. The occiput to wall distance test and Schober's test were negative. The results of the immunological tests, including antinuclear antibodies, rheumatoid factor, and anti-cyclic citrullinated peptide antibodies, were negative. Human leukocyte antigen (HLA) typing was positive for HLA B27. Radiological examinations, including pelvis CT, showed sacroiliitis grade III bilaterally (Fig. 2). A diagnosis of AS was made by the modified New York criteria. According to the Assessment Spondyloarthritis International Society criteria, clinical findings such as inflammatory back pain over 3 months, sacroiliitis, knee arthritis, anterior uveitis and HLA B27 positivity represented spondyloarthritis although her age did not meet the criteria. At the time of diagnosis, serum levels of erythrocyte sedimentation rate $(22 \mathrm{~mm} / \mathrm{hr})$ and C-reactive protein (1.2 mg/L) were normal range, but the Bath Ankylosing Spondylitis Disease Activity Index (BASDAI) was 6.4. She was commenced nonsteroidal anti-inflammatory drugs (NSAIDs) and sulfasalazine with an increasing dose (50 mg) of spironolactone. Refractory to treatment with NSAIDs and sulfasalazine, we decided to start TNF- $\alpha$ inhibitor therapy. She rapidly responded to the treatment and BASDAI decreased from 6.4 to 2.0 after 3 months therapy.
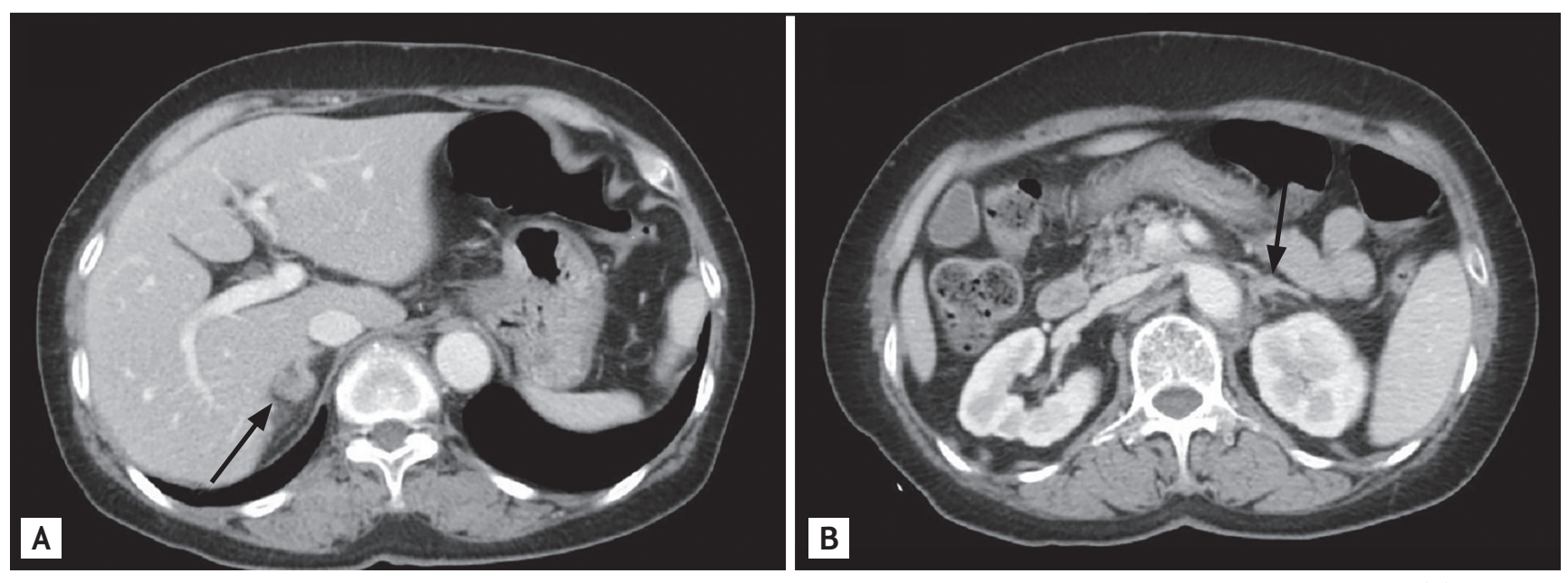

Figure 1. Computed tomography of the abdomen: the arrows show two $20 \mathrm{~mm}$ nodules in the right adrenal gland (A) and a 10 $\mathrm{mm}$ nodule in the left adrenal gland (B). 


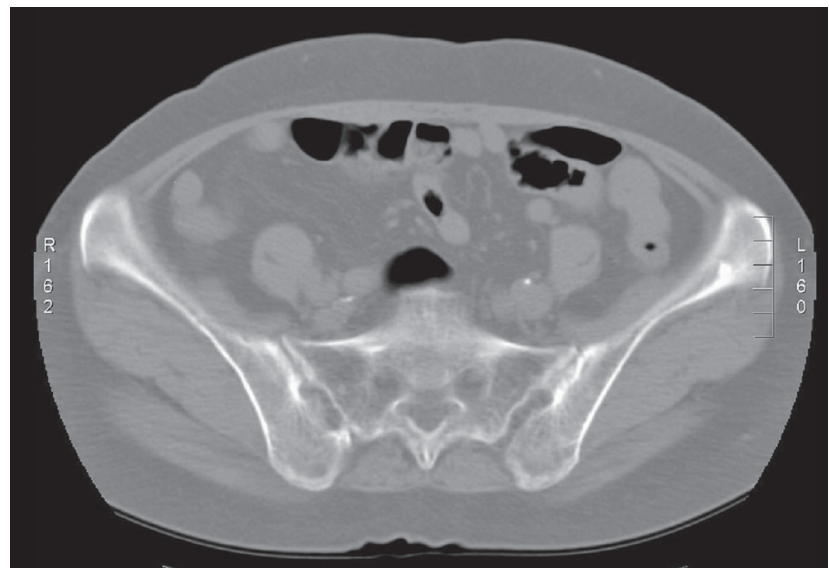

Figure 2. Computed tomography of the pelvis shows intraarticular bony ankylosis and erosion with pseudo-widening in both sacroiliac joints.

To our knowledge, this report is the first to describe the coexistence of primary aldosteronism and AS in a middle-aged woman. Whether these two diseases developed in the same patient accidentally cannot be excluded, but these diseases were thought to be related to each other. Until now there has been no literature about direct association of primary aldosteronism with spondyloarthropathy. However, increasing evidence has shown that innate and adaptive immune responses are modulated by aldosterone, which promotes inflammatory cytokine production such as TNF- $\alpha$. In addition, the observation that aldosterone can promote $\mathrm{CD}_{4}+\mathrm{T}$ cell activation and Th17 polarization suggests that this hormone could contribute to the onset of autoimmunity [3]. TNF- $\alpha$ and interleukin (IL) 17 are also important cytokine in spondyloarthritis [1]. Therefore, we thought that primary aldosteronism may stimulate or worsen autoimmune inflammatory diseases.

Actually, there are several reports regarding an association between primary aldosteronism and autoimmune diseases such as autoimmune thyroiditis. Tanaka et al. [4] described a case of combined primary aldosteronism and Hashimoto's thyroiditis. Krysiak and Okopien [5] demonstrated that excessive aldosterone release may lead to the development of thyroid infiltration by inflammatory cells and an elevation of proinflammatory cytokines, such as TNF- $\alpha$ in the presence of an aldosterone-producing adrenal tumor. After spironolactone pretreatment and surgery, the levels of proinflammatory cytokines were reduced and thyroid function was im- proved [5]. Although we did not check the production of proinflammatory cytokines, we assumed that excessive aldosterone induced the release of more proinflammatory cytokines and affected the development of AS in our patient.

Laparoscopic adrenalectomy is currently the best treatment. However, for patients who are not candidates for surgery or do not show lateralized aldosterone excess, mineralocorticoid receptor antagonists, such as spironolactone, are a reasonable alternative to adrenalectomy [1]. In this patient, lateralized aldosterone secretion was not demonstrated by AVS. Thus, she was given spironolactone and had effectively controlled blood pressure and electrolytes balance. The recent report showed that primary aldosteronism patients display increased secretion of TNF- $\alpha$, transforming growth factor beta, and IL-10 compared to controls, and spironolactone only partially restored these levels [3]. Therefore, spironolactone might influence AS treatment. However, that effect was insignificant in our patient, so she also needed a TNF- $\alpha$ inhibitor to control her AS.

Our report supports that primary aldosteronism could be related to chronic inflammatory diseases due to its proinflammatory effects. Therefore, we should pay attention to the possibility of chronic inflammatory autoimmune diseases, such as AS, in patients with primary aldosteronism.

Keywords: Hyperaldosteronism; Spondylitis, ankylosing

\section{Conflict of interest}

No potential conflict of interest relevant to this article was reported.

\section{REFERENCES}

1. Rossi GP. A comprehensive review of the clinical aspects of primary aldosteronism. Nat Rev Endocrinol 2011;7:485-495.

2. Dougados M, Baeten D. Spondyloarthritis. Lancet 2011; 377:2127-2137.

3. Herrada AA, Campino C, Amador CA, Michea LF, Fardella CE, Kalergis AM. Aldosterone as a modulator of immunity: implications in the organ damage. J Hypertens 2011;29:1684-1692. 
4. Tanaka M, Izeki M, Miyazaki Y, et al. Combined primary aldosteronism and Cushing's syndrome due to a single adrenocortical adenoma complicated by Hashimoto's thyroiditis. Intern Med 2002;41:967-971.
5. Krysiak R, Okopien B. Coexistence of primary aldosteronism and Hashimoto's thyroiditis. Rheumatol Int 2012;32:2561-2563. 